\title{
Phytochemical and cytotoxic investigations of the lichen Stereocaulon evolutum Graewe
}

\author{
Vu Thi Huyen ${ }^{1,5}$, Nguyen Thi Thu Tram ${ }^{2,5 *}$, Nguyen Manh Cuong ${ }^{3}$, \\ Nguyen Phuc Dam ${ }^{4}$, Lohézic-Le Dévéhat Françoise ${ }^{5}$, Boustie Joël ${ }^{5}$ \\ ${ }^{I}$ Department of Chemistry, Faculty of Environment, Vietnam National University of Agriculture \\ ${ }^{2}$ Department of Chemistry, Faculty of Science, Can Tho University of Medicine and Pharmacy \\ ${ }^{3}$ Institute of Natural Products Chemistry, Vietnam Academy of Science and Technology \\ ${ }^{4}$ Department of Chemistry, School of Education, Can Tho University \\ ${ }^{5}$ Team PNSCM, UMR CNRS 6226, Faculty of Pharmacy, University of Rennes 1, France
}

Received 9 January 2017; Accepted for publication 28 August 2017

\begin{abstract}
Lichens are fungal and algal/cyanobacterial symbioses resulting in the production of specific metabolites with a great variety of effects such as antibiotic, antimycobacterial, antiviral, anti-inflammatory, analgesic, antipyretic and antiproliferative. As a part of searching cytotoxic compounds from lichens, a phytochemical investigation was conducted on a squamulose lichen, Stereocaulon evolutum Graewe, collected in France. Five known compounds including atranorin (1), atranol (2), lobaric acid (3), ursolic acid (4), and methyl 3'-methyllecanorate (5) were isolated for the first time from such lichen. Their structures were elucidated by ESI-HR-MS, NMR spectral analysis and compared with literature data. Cytotoxicity screening of five isolated compounds were evaluated on seven cell lines including $\mathrm{HuH} 7$, CaCo-2, MDA-MB-231, HCT116, PC3, NCI-H727 and HaCaT. Compound (5) was found high cytotoxicity effect on all investigated cell lines while other compounds showed non-cytotoxic.
\end{abstract}

Keywords. Lichen, Stereocaulon, atranorin, cytotoxicity.

\section{INTRODUCTION}

Lichens are stable, consistent and identifiable mutualistic associations between algae and/or cyanobacteria and fungi [1]. About 1035 secondary metabolites have been isolated from the 18500 lichen species described to date, and many more compounds remain to be characterized. Most of these compounds are polyketides, polyphenols, quinones or terpenoids, presumably of fungal origin and their biological activities remain largely underexplored [2, 3]. However, a few have been shown to have antibiotic, antimycobacterial, antiviral, anti-inflammatory, analgesic, antipyretic, antiproliferative and cytotoxic activities [3] Stereocaulon is a widely distributed worldwide lichen genus with around 130 species but approximate 40 species were investigated phytochemistry due to their difficult identification. Among 75 isolated substances from Stereocaulon, atranorin is a constant compound as well as the common occurrence of three depsidones including lobaric acid, stictic acid and norstictic acid is useful for taxonomy in such genus [4]. Various isolated lichen compounds often demonstrate significant inhibitory activity against several cancer cell lines at very low concentrations.

Although lichens are a source for excellent anticancer active compounds, only a small number have been tested for their biological significance [5]. In this paper, as a part of our searching cytotoxic compounds from lichens, a phytochemical investigation was conducted on a squamulose lichen, Stereocaulon evolutum Graewe, collected in France. Atranorin (1), a major compound, along with four other compounds atranol (2), lobaric acid (3), ursolic acid (4) and methyl 3'-methyllecanorate (5) were isolated. Their cytotoxic activity was evaluated on seven cell lines $\mathrm{HuH} 7, \mathrm{CaCo}-2$, MDA-MB-231, HCT116, PC3, NCI-H727 and HaCaT. 


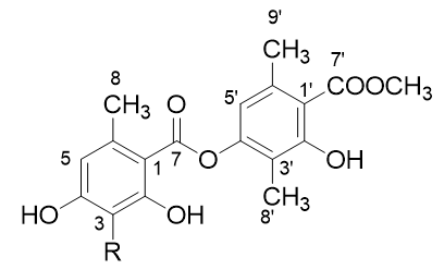

Atranorin $(\mathrm{R}=\mathrm{CHO})(\mathbf{1})$

Methyl 3'-methyllecanorate $(\mathrm{R}=\mathrm{H})(\mathbf{5})$

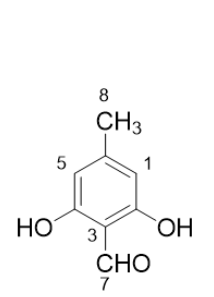

Atranol (2)

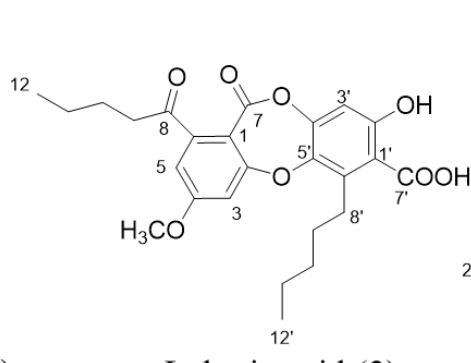

Lobaric acid (3)

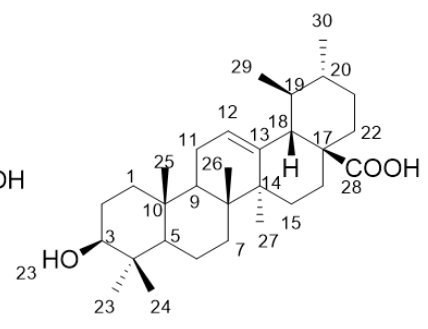

Ursolic acid (4)

Figure 1: Structures of compounds isolated from the lichen S. evolutum

\section{MATERIALS AND METHODS}

\subsection{General procedures}

All solvents for chromatography were purchased from Sigma-Aldrich (France). Thin-layer chromatography (TLC) was carried out on silica gel plates (Merck silica gel 60F254) with the A (toluene:dioxane:acetic acid 180:45:5), B (nhexane:diethyl ether:formic acid 130:80:20), and C (toluene:acetic acid 170:30) standard solvent systems for the identification of substances from lichens [6]. The spots were first visualized under UV light and then after spraying with anisaldehyde$\mathrm{H}_{2} \mathrm{SO}_{4}$ reagent. Circular chromatography and column chromatography were carried out on silica gel (40-63 $\mu \mathrm{m}$, Kieselgel 60, Merck, 7667). Flash column chromatography was performed on a SPOT Flash liquid chromatography (Armen Instrument), using RP-18 columns (Chromabond, Merck). The NMR experiments were performed on a Bruker DMX 300 and 500 spectrometers with deuterated solvents. High-resolution mass spectrometry (HRMS) measurements were carried out to determine exact mass, on a Bruker Maxis 4G, MicrO-Tof Q 2, a Thermo-fisher Q-Exactive or a Waters Q-Tof 2 mass spectrometer for chemical ionization. Melting points were measured on a Kofler LEICA VMHB.

\subsection{Lichen materials}

Stereocaulon evolutum Graewe was collected in Bretagne, France, on November 2011. The scientific name was identified by $\mathrm{PhD}$ Lohézic-Le Dévéhat Françoise, Faculty of Pharmacy, University of Rennes 1, France. A voucher specimen (JB/10/121) was deposited in the herbarium of Pharmacognosy and Mycology, University of Rennes 1, France.

\subsection{Cytotoxicity assay}

The cell lines were obtained from the ECACC collection. Cells were grown according to ECACC recommendations. The toxicity test of the compounds on these cells was as follows: 2.103 cells/well for HCT116 cell line or 4.103 cells/well for the other cell lines were seeded in 96 well plates. After $24 \mathrm{~h}$ of seeding, cells were exposed to the compounds at concentration $10 \mu \mathrm{M}$. After $48 \mathrm{~h}$ of treatment, the cells were washed in PBS and fixed in ethanol/acetic acid (90:5 v:v) for $20 \mathrm{~min}$. Then, the nuclei were stained with Hoechst 3342 (Sigma). Image acquisition and analysis was performed using a Cellomics ArrayScan VTI/HCS Reader (Thermo Scientific).

\subsection{Extraction and isolation}

Air-dried crushed thalli of the lichen S. evolutum Graewe $(300 \mathrm{~g})$ were successively extracted with $n$ hexane, acetone and tetrahydrofuran, by maceration $24 \mathrm{~h}$ at room temperature ( 3 times $\times 2 \mathrm{~L}$ ). A pure colorless compound 1 (6.2 g) was precipitated from the $n$-hexane and acetone extracts by evaporating off the solvents at room temperature. The $n$-hexane filtrate $(1.5 \mathrm{~g})$ was fractionated on successive silica gel columns and then circular chromatography using $n$-hexane:dichloromethane 3:7 to afford compound $\mathbf{4}$ $(9.0 \mathrm{mg})$. The acetone filtrate $(13.4 \mathrm{~g})$ was subjected to silica gel flash column chromatography (Chromabond $^{\circledR}$ flash, RS 40 C18) using a water:acetonitrile gradient system $(9: 1,8: 2,7: 3,6: 4$, 5:5, 4:6, 0:1, each $225 \mathrm{~mL}$ ) to give seven fractions Ac1-Ac7. A precipitate in fraction Ac6 was filtrated and recrystallization in acetone to yield compound $\mathbf{3}$ $(1.2 \mathrm{~g})$. Fraction Ac7 was purified by a silica gel column chromatography using $n$-hexane:ethyl acetate $8: 2$ to give compound $2(10.0 \mathrm{mg})$ and compound 5 (21 mg).

Atranorin (1): colorless powder; $\mathrm{R}_{\mathrm{f}}=0.78(\mathrm{~B})$, $\mathrm{R}_{\mathrm{f}}=0.70(\mathrm{C}) ;$ M.p $196-197{ }^{\circ} \mathrm{C} ;{ }^{1} \mathrm{H}$ NMR (DMSO$\left.d_{6}, 500 \mathrm{MHz}\right): \delta_{\mathrm{H}} 10.52(1 \mathrm{H}, s, 2-\mathrm{O} \underline{\mathbf{H}}), 10.21(1 \mathrm{H}, s$, 3-CHO), 6.41 (1H, $s, \mathrm{H}-5), 2.35$ (3H, $s, \mathrm{H}-8), 6.65$ $\left(1 \mathrm{H}, s, \mathrm{H}-5\right.$ '), $2.04\left(3 \mathrm{H}, s, \mathrm{H}-8^{\prime}\right), 2.39\left(3 \mathrm{H}, s, \mathrm{H}-9^{\prime}\right)$, $3.88\left(3 \mathrm{H}, s, 77^{-}-\mathrm{OC}_{3}\right) ;{ }^{13} \mathrm{C}$ NMR (DMSO- $d_{6}, 125$ $\mathrm{MHz}) \delta_{\mathrm{C}}: 107.9(\mathrm{C}-1), 163.6(\mathrm{C}-2), 109.0(\mathrm{C}-3)$, 
161.7 (C-4), 115.2 (C-5), 151.4 (C-6), 164.5 (C-7), 193.8 (C-8), 20.1 (C-9), 110.6 (C-1'), 157.4 (C-2'), 116.3 (C-3'), 148.8 (C-4'), 115.7 (C-5'), 136.5 (C-6'), 169.7 (C-7'), 9.3 (C-8'), 21.1 (C-9'), $52.3\left(\right.$ (7'-OCH $\left._{3}\right)$; ESI-HRMS $\mathrm{m} / \mathrm{z} 397.0899 \quad[\mathrm{M}+\mathrm{Na}]^{+}$(calcd. for $\mathrm{C}_{19} \mathrm{H}_{18} \mathrm{O}_{8}$ ).

Atranol (2): yellow needle (in $\mathrm{CHCl}_{3}$ ); $\mathrm{R}_{\mathrm{f}}=0,70$ (C); M.p $124{ }^{\circ} \mathrm{C} ;{ }^{1} \mathrm{H}$ NMR (acetone- $d_{6}, 500 \mathrm{MHz}$ ): $\delta_{\mathrm{H}} 10.69(1 \mathrm{H}, s, 2-\mathrm{O} \underline{\mathbf{H}}), 10.69(1 \mathrm{H}, s, 4-\mathrm{OH}), 10.27$ $(1 \mathrm{H}, s, \mathrm{H}-7), 6.26(1 \mathrm{H}, s, \mathrm{H}-1), 6.26(1 \mathrm{H}, s, \mathrm{H}-5)$, $2.23(3 \mathrm{H}, s, \mathrm{H}-8) ;{ }^{13} \mathrm{C}$ NMR (acetone- $\left.d_{6}, 125 \mathrm{MHz}\right)$ $\delta_{\mathrm{C}}: 108.4(\mathrm{C}-1), 163.0(\mathrm{C}-2), 109.2(\mathrm{C}-3), 163.0(\mathrm{C}-$ 4), 108.4 (C-5), 151.5 (C-6), 194.1 (C-7), 22.2 (C8); ESI-HRMS $\mathrm{m} / z 175.0373[\mathrm{M}+\mathrm{Na}]^{+}$(calcd. for $\mathrm{C}_{8} \mathrm{H}_{8} \mathrm{O}_{3}$ ).

Lobaric acid (3): white crystal (in acetone); $\mathrm{R}_{\mathrm{f}}=$ 0.46 (B), $\mathrm{R}_{\mathrm{f}}=0.41$ (C); M.p 196-197 ${ }^{\circ} \mathrm{C} ;{ }^{1} \mathrm{H}$ NMR (acetone- $\left.d_{6}, 500 \mathrm{MHz}\right): \delta_{\mathrm{H}} 7.00(1 \mathrm{H}, d, 2.2, \mathrm{H}-3)$, $7.00(1 \mathrm{H}, d, 2.2, \mathrm{H}-5), 6.74(1 \mathrm{H}, s, \mathrm{H}-3$ '), $3.98(3 \mathrm{H}$, $\left.s, 4-\mathrm{OC}_{3}\right), 3.30(2 \mathrm{H}, t, 5.5, \mathrm{H}-8$ '), $2.85(2 \mathrm{H}, t, 7.7$, H-9), 1.63 (2H, $m, \mathrm{H}-10), 1.63$ (2H, $\left.m, \mathrm{H}-9{ }^{\prime}\right), 1.51$ $\left(2 \mathrm{H}, m, \mathrm{H}-10^{\prime}\right), 1.39$ (2H, $\left.m, \mathrm{H}-11\right), 1.39(2 \mathrm{H}, m, \mathrm{H}-$ $\left.11^{\prime}\right), 0.91(2 \mathrm{H}, m, \mathrm{H}-12), 0.91\left(2 \mathrm{H}, m, \mathrm{H}-12^{\prime}\right) ;{ }^{13} \mathrm{C}$ NMR (acetone- $\left.d_{6}, 125 \mathrm{MHz}\right) \delta_{\mathrm{C}}: 112.9(\mathrm{C}-1), 163.9$ (C-2), 106.9 (C-3), 165.5 (C-4), 112.2 (C-5), 150.2 (C-6), 161.0 (C-7), 203.4 (C-8), 42.3 (C-9), 26.7 (C10), 23.2 (C-11), 14.2 (C-12), 112.2 (C-1'), 162.5 (C-2'), 107.8 (C-3'), 148.9 (C-4'), 142.3 (C-5'), 139.9 (C-6'), 172.4 (C-7'), 28.8 (C-8'), 32.0 (C-9'), 33.0 (C10'), 22.8 (C-11'), 14.4 (C-12'), $56.9\left(4-\mathrm{OCH}_{3}\right)$; ESIHRMS $m / z 455.1713$ [M-H] ' (calcd. for $\mathrm{C}_{25} \mathrm{H}_{28} \mathrm{O}_{8}$ ).

Ursolic acid (4): white solid; $\mathrm{R}_{\mathrm{f}}=0.50(\mathrm{~B}), \mathrm{R}_{\mathrm{f}}=$ 0.49 (C); M.p $290-292{ }^{\circ} \mathrm{C} ;{ }^{1} \mathrm{H}$ NMR $\left(\mathrm{CD}_{3} \mathrm{OD}, 300\right.$ $\mathrm{MHz}): \delta_{\mathrm{H}} 5.23(1 \mathrm{H}, \mathrm{t}, 3.5, \mathrm{H}-12), 3.16(1 \mathrm{H}, \mathrm{dd}, 6.0$, 6.0, H-3), $2.22(1 \mathrm{H}, \mathrm{bd}, \mathrm{H}-18), 1.12,0.98,0.96$, 0.90, 0.87, 0.85, $0.78(21 \mathrm{H}, 7 \times \mathrm{s}, 7 \times-\mathrm{Me}) .{ }^{13} \mathrm{C} \mathrm{NMR}$ $\left(\mathrm{CD}_{3} \mathrm{OD}, 75 \mathrm{MHz}\right) \delta_{\mathrm{C}}: 39.8(\mathrm{C}-1), 27.9(\mathrm{C}-2), 79.7$ (C-3), 40.4 (C-4), 56.7 (C-5), 19.5 (C-6), 34.3 (C-7), 40.8 (C-8), 48.1 (C-9), 38.1 (C-10), 24.1 (C-11), 126.8 (C-12), 139.6 (C-13), 43.2 (C-14), 28.7 (C15), 25.3 (C-16), 48.1 (C-17), 54.4 (C-18), 40.4 (C19), 40.0 (C-20), 31.8 (C-21), 38.1 (C-22), 28.7 (C23), 16.4 (C-24), 16.0 (C-25), 17.6 (C-26), 24.3 (C27), 181.7 (C-28), 17.8 (C-29), 21.6 (C-30); ESIHRMS $m / z$ 479.3499 [M+Na] ${ }^{+}$(calcd. for $\mathrm{C}_{30} \mathrm{H}_{48} \mathrm{O}_{3}$ ).

Methyl 3'-methyllecanorate (5): white crystal (in acetone); $\mathrm{R}_{\mathrm{f}}=0.55(\mathrm{~A}), \mathrm{R}_{\mathrm{f}}=0.40(\mathrm{C}) ;$ M.p 126-128 ${ }^{\circ} \mathrm{C} ;{ }^{1} \mathrm{H}$ NMR $\left(\mathrm{CDCl}_{3}, 300 \mathrm{MHz}\right): \delta_{\mathrm{H}} 11.43(1 \mathrm{H}, s, 2-$ $\mathrm{OH}), 11.96(1 \mathrm{H}, s, 2-\mathrm{OH}), 6.53\left(1 \mathrm{H}, s, \mathrm{H}-5^{\prime}\right), 6.63$ $(1 \mathrm{H}, d, 2.58, \mathrm{H}-3), 6.63(1 \mathrm{H}, d, 2.58, \mathrm{H}-5), 5.47(1 \mathrm{H}$, $s$, 4-OH), $3.99\left(3 \mathrm{H}, s, 7-\mathrm{OCH}_{3}\right) 2.65(3 \mathrm{H}, s, \mathrm{H}-8)$, $2.54\left(3 \mathrm{H}, s, \mathrm{H}-9^{\prime}\right), 2.10\left(3 \mathrm{H}, s, \mathrm{H}-8^{\prime}\right) ;{ }^{13} \mathrm{C} \mathrm{NMR}$ $\left(\mathrm{CDCl}_{3}, 75 \mathrm{MHz}\right) \delta_{\mathrm{C}}: 104.5(\mathrm{C}-1), 166.3(\mathrm{C}-2)$, 101.5 (C-3), 161.1 (C-4), 111.9 (C-5), 144.4 (C-6), 169.7 (C-7), 24.6 (C-8), 109.9 (C-1'), 162.8 (C-2'),
116.8 (C-3'), 152.3 (C-4'), 116.2 (C-5'), 139.7 (C-6'), 172.2 (C-7'), 9.3 (C-8'), $24.0\left(\mathrm{C}-9^{\prime}\right), 52.3\left(7^{\prime}-\mathrm{OCH}_{3}\right)$; ESI-HRMS $\mathrm{m} / z \quad 345.0979 \quad[\mathrm{M}-\mathrm{H}]^{-}$(calcd. for $\mathrm{C}_{18} \mathrm{H}_{18} \mathrm{O}_{7}$ ).

\section{RESULTS AND DISCUSSION}

The five known compounds atranorin $(\mathbf{1}, 6.2 \mathrm{~g})$, atranol $(2,10 \mathrm{mg})$, lobaric acid $(\mathbf{3}, 1.2 \mathrm{~g})$, ursolic acid $(\mathbf{4}, 9.0 \mathrm{mg})$ and methyl 3'-methyllecanorate $(\mathbf{5}$, $21 \mathrm{mg}$ ) were identified by direct comparison of their physical and spectral data in the literature $[6,7]$. Although the compounds have previously been described in some others species of genus Stereocaulon [4], here, they were reported for the first time in Stereocaulon evolutum (figure 1).

The cytotoxic activity of the five isolated compounds was performed on seven cell lines at concentration of $10 \mu \mathrm{M}$. The results showed that no cytotoxicity was observed in almost investigated compounds, except $\mathbf{5}$. In fact, the percentage of cell viability was over $80 \%$ for $\mathbf{1}, \mathbf{2}, \mathbf{3}$ and $\mathbf{4}$ while this value of $\mathbf{5}$ is $52 \%, 30 \%, 66 \%, 17 \%, 35 \%, 69 \%$ and $27 \%$ on $\mathrm{HuH} 7$, Caco-2, MDA-MB231, HCT116, PC3, NCI-H727 and HaCaT, respectively (table 1). Compound $\mathbf{5}$ displayed higher toxicity than atranorin (1) which possesses an aldehyde functional group on C-3 which is well known responsible to apoptosis [8]. It is worth nothing that the screening results were not opposite to previous reports [4] since the investigated compounds showed cytotoxic activities in dose much higher than $10 \mu \mathrm{M}$.

Acknowledgments. We thank Vietnamese Government (322 project) for a PhD grant to VTH.

\section{REFERENCES}

1. V. Shukla, D. K. Upreti and R. Bajpai. Lichens to biomonitor the environment, Springer India (2014).

2. E. Stocker-Wörgötter. Metabolic diversity of lichenforming ascomycetous fungi: culturing, polyketide and shikimate metabolite production, and PKS genes, Nat. Prod. Rep., 25, 188-200 (2008).

3. J. Boustie and M. Grube. Lichens-a promising source of bioactive secondary metabolites, Plant Genet. Resour., 3(2), 273-287 (2005).

4. T. H. Vu. Etude des acides gras du genre Stereocaulon, Etude phytochimique du lichen $S$. evolutum Graewe, Ph.D thesis, Rennes 1 University, France (2014).

5. R. Branislav. Lichen secondary metabolites. Bioactive properties and pharmaceutical potential, 127-142, Springer (2015).

6. S. Huneck and I. Yoshimura. Identification of lichen 
substances, Springer Verlag, Berlin (1996).

7. T.H. Vu, A-C. Le Lamer, C. Lalli, J. Boustie, M. Samson, F. Lohézic-Le Dévéhat and J. Le Seyec. Depsides, lichen metabolites active against Hepatitis C virus, PLoS One, 10(3) (2015).
8. M. Bačkorová, R. Jendželovský, M. Kello, M. Bačkor, J. Mikeš and P. Fedoročko. Lichen secondary metabolites are responsible for induction of apoptosis in HT-29 and A2780 human cancer cell lines, Toxicol in Vitro, 26, 462-468 (2012).

Table 1: Cytotoxicity of compounds $\mathbf{1}, \mathbf{2}, \mathbf{3}, \mathbf{4}$, and $\mathbf{5}$ against seven cell lines at $\mathrm{C}=10 \mu \mathrm{M}$

\begin{tabular}{|l|c|c|c|c|c|c|c|}
\hline \multirow{2}{*}{ Cell lines } & \multicolumn{7}{c|}{ Cell viability (\%) } \\
\cline { 2 - 9 } Compounds & HuH7 & Caco-2 & MDA-MB231 & HCT116 & PC3 & NCI-H727 & HaCaT \\
\hline Atranorin (1) & 98 & 109 & 85 & 107 & 101 & 82 & 97 \\
\hline Atranol (2) & 94 & 125 & 100 & 103 & 103 & 91 & 102 \\
\hline Lobaric acid (3) & 96 & 123 & 87 & 102 & 88 & 93 & 92 \\
\hline Ursolic acid (4) & 104 & 111 & 106 & 122 & 105 & 108 & 115 \\
\hline $\begin{array}{l}\text { Methyl 3'-methyl lecanorate } \\
(\mathbf{5})\end{array}$ & $\mathbf{5 2}$ & $\mathbf{3 0}$ & $\mathbf{6 6}$ & $\mathbf{1 7}$ & $\mathbf{3 5}$ & $\mathbf{6 9}$ & $\mathbf{2 7}$ \\
\hline
\end{tabular}

Note: Huh7 (human hepato cellular carcinoma), CaCo-2 (human colorectal adenocarcinoma), MDA-MB-231 (human breast cancer), HCT116 (human colon cancer), PC3 (human prostate cancer), NCI-H727 (human lung cancer), HaCaT (human immortalized keratinocytes).

\section{Corresponding author: Nguyen Thi Thu Tram}

Can Tho University of Medicine and Pharmacy

No. 179, Nguyen Van Cu Str., An Khanh Ward, Ninh Kieu Dist., Can Tho City

E-mail: ntttram@ctump.edu.vn; Telephone: 0919886682. 